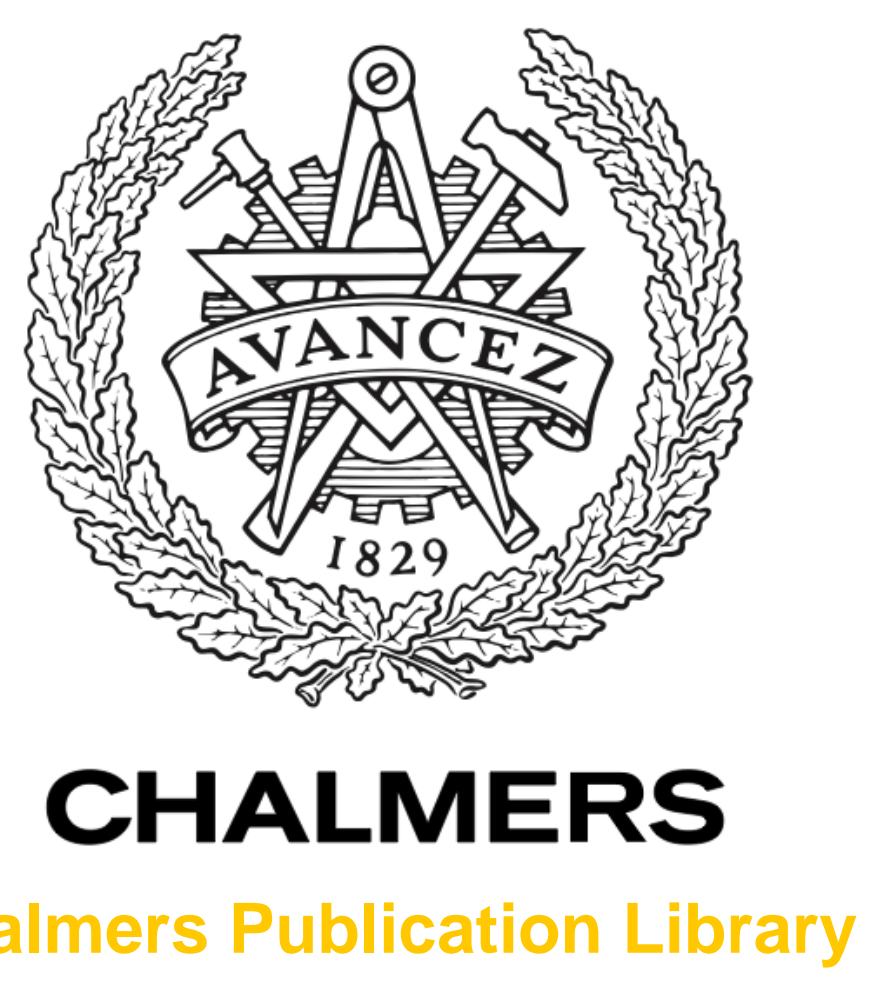

Chalmers Publication Library The Method of Equivalent Dipole Moments (MEDM) - a Novel CBFM Approach for
the Fast and Accurate Solution of Dielectric-Scattering Problems

This document has been downloaded from Chalmers Publication Library (CPL). It is the author's version of a work that was accepted for publication in:

ICEAA-IEEE APWC, 12-17 September 2011 in Torino, Italy

Citation for the published paper:

Naeem, M. ; Maaskant, R. ; kant, D. (2011) "The Method of Equivalent Dipole Moments (MEDM) - a Novel CBFM Approach for the Fast and Accurate Solution of DielectricScattering Problems". ICEAA-IEEE APWC, 12-17 September 2011 in Torino, Italy

Downloaded from: http://publications.lib.chalmers.se/publication/153391

Notice: Changes introduced as a result of publishing processes such as copy-editing and formatting may not be reflected in this document. For a definitive version of this work, please refer to the published source. Please note that access to the published version might require a subscription.

Chalmers Publication Library (CPL) offers the possibility of retrieving research publications produced at Chalmers University of Technology. It covers all types of publications: articles, dissertations, licentiate theses, masters theses, conference papers, reports etc. Since 2006 it is the official tool for Chalmers official publication statistics. To ensure that Chalmers research results are disseminated as widely as possible, an Open Access Policy has been adopted.

The CPL service is administrated and maintained by Chalmers Library. 


\title{
The Method of Equivalent Dipole Moments (MEDM) Combined with CBFM for the Fast and Accurate Solution of Dielectric Scattering Problems
}

\author{
M. Naeem* \\ R. Maaskant ${ }^{\dagger}$ \\ G. W. Kant ${ }^{\ddagger}$ \\ P.-S. Kildal ${ }^{\S}$ \\ R. Mittra
}

\begin{abstract}
High-resolution basis functions are employed for accurate modelling of electromagnetic scattering from dielectric objects. It is shown that the field radiated by each of these micro basis functions resembles the dipole field, even in the immediate vicinity of its cubic support. This, in turn, significantly eases the computational burden of generating the off-diagonal elements of the moment matrix. Furthermore, the on-diagonal self-term is known analytically and only governed by the electrostatic field (electrodynamic part is negligible). This method has been hybridised with the Characteristic Basis Function Method (CBFM) and the Adaptive Cross Approximation (ACA) algorithm to reduce both the size and generation time of the moment matrix equation. It is demonstrated that the proposed method, herein referred to as MEDM, is not only fast and memory efficient but it also generates an accurate solution of scattering problems associated with complex-shaped, thin and electrically large objects.
\end{abstract}

\section{INTRODUCTION}

In various low-loss $\mathrm{RF}$ applications, it is important to evaluate the electromagnetic scattering and power dissipation losses accurately [1], for instance in dielectric objects such as radomes for radio astronomy. However, the numerical analysis becomes a burdensome task for most commercially available CEM tools if the dielectric is thin, complex-shaped, and electrically large.

Taking a cue from [2], we propose to employ socalled micro-domain basis functions - for modelling the volume equivalent current - that are one-to-two order smaller in size than conventional basis functions. The proposed basis functions can approximate the boundaries of arbitrary-shaped geometries accurately by representing them with a cubic volume of uniform current. Furthermore, since each micro-domain basis function is electrically small,

\footnotetext{
*Department of Signals and Systems, Chalmers Univ. of Technology, Sweden, e-mail: naeem@astron.nl.

${ }^{\dagger}$ Department of Signals and Systems, Chalmers Univ. of Technology, Sweden, e-mail: rob.maaskant@chalmers.se.

${ }^{\ddagger}$ Netherlands Institute for Radio Astronomy, The Netherlands, e-mail: kant@astron.nl.

$\S$ Department of Signals and Systems, Chalmers Univ. of Technology, Sweden, e-mail: per-simon.kildal@chalmers.se.

"EMC Lab, EE Department, Pennsylvania State Univ., USA, e-mail: mittra@engr.psu.edu.
}

the field that it generates closely resembles that of the dipole field. This field is known in closed form and can be tested by using the collocation method, and by employing the basis functions in the adjacent cells, since these also have an electrically small support. Finally, the field tested over its own support can be computed in closed form by retaining just their quasi-static contributions.

The major drawback of this approach, however, is that the number of basis functions increases dramatically in comparison with that when the conventional bases are employed. To mitigate this problem, we first employ the CBFM, [3], which reduces the number of independent basis functions significantly, and then hybridise it with the ACA algorithm for rapid construction of the reduced matrix [4].

The resulting numerical scheme is easy to implement; yet it is accurate, and can handle large problems of dielectric contrast, in particular in a multilevel context (MLCBFM). In effect, the Characteristic Basis Functions (CBFs) are constructed from low-level basis functions, so that one can view this method as being equivalent to employing higherorder (conformal) basis functions of conventional size in conjunction with an accurate integration scheme. However, the advantage of the present method is that it is much simpler. We begin by in-

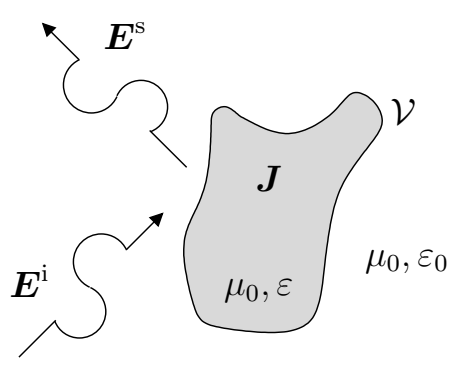

Figure 1: The EM scattering from a dielectric object of arbitrary shape.

troducing the concept of the method of equivalent dipole moments (MEDM). Next, we describe the CBFM and show the results for a canonical problem of scattering by a dielectric sphere to test the numerical accuracy, convergence, and the compu- 
tational efficiency. Finally, we compare our results with those obtained from the commercially available CEM solver, Ansoft HFSS, as well as with the Mie series solution, which is available analytically.

\section{The Method of Equivalent Dipole Mo- ments (MEDM)}

Figure 1 illustrates a dielectric object with volume $\mathcal{V}$, permittivity $\varepsilon$, and a permeability $\mu$ identical to that of free space, $\mu_{0}$. The object is illuminated by an electromagnetic field $\boldsymbol{E}^{\mathrm{i}}$. After invoking the volume equivalence principle, the object is replaced by the surrounding medium (vacuum), while the equivalent electric current $\boldsymbol{J}_{\text {eq }}$ inside the object gives rise to the original scattered field $\boldsymbol{E}^{\mathrm{s}}\left(\boldsymbol{J}_{\mathrm{eq}}\right)$, such that the field consistency condition $\boldsymbol{J}_{\text {eq }}=j \omega\left(\varepsilon-\varepsilon_{0}\right) \boldsymbol{E}$, for $\boldsymbol{r} \in \mathcal{V}$ holds, where $\boldsymbol{E}=\boldsymbol{E}^{\mathrm{i}}+\boldsymbol{E}^{\mathrm{S}}\left(\boldsymbol{J}_{\mathrm{eq}}\right)$, and

$$
\boldsymbol{E}^{\mathrm{S}}\left(\boldsymbol{r}, \boldsymbol{J}_{\mathrm{eq}}\right)=-j \omega \mu_{0} \boldsymbol{A}\left(\boldsymbol{r}, \boldsymbol{J}_{\mathrm{eq}}\right)-\nabla \Phi\left(\boldsymbol{r}, \boldsymbol{J}_{\mathrm{eq}}\right)
$$

where $\boldsymbol{A}$ and $\Phi$ are the electric vector and scalar potentials, respectively. These can be expressed as

$$
\begin{aligned}
& \boldsymbol{A}=\iiint_{\mathcal{V}} G\left(\boldsymbol{r}-\boldsymbol{r}^{\prime}\right) \boldsymbol{J}_{\mathrm{eq}}\left(\boldsymbol{r}^{\prime}\right) \mathrm{d} V^{\prime}, \\
& \Phi=\frac{-1}{j \omega \varepsilon_{0}} \iiint_{\mathcal{V}} G\left(\boldsymbol{r}-\boldsymbol{r}^{\prime}\right) \boldsymbol{\nabla} \cdot \boldsymbol{J}_{\mathrm{eq}}\left(\boldsymbol{r}^{\prime}\right) \mathrm{d} V^{\prime},
\end{aligned}
$$

where $G$ is the scalar free-space Green's function given by $\exp \left(-j k_{0}\left|\boldsymbol{r}-\boldsymbol{r}^{\prime}\right|\right) /\left(4 \pi\left|\boldsymbol{r}-\boldsymbol{r}^{\prime}\right|\right)$. In common with the conventional MoM discretisation, we proceed in MEDM by expanding the volume equivalent current as $\boldsymbol{J}_{\text {eq }}=\sum_{n=1}^{N} I_{n} \boldsymbol{f}_{n}(\boldsymbol{r})$, where $\left\{\boldsymbol{f}_{n}\right\}_{n=1}^{N}$ are the $N$ micro-basis functions, and $\left\{I_{n}\right\}_{n=1}^{N}$ are the corresponding expansion coefficients. Next, we use the collocation method to test the field at the centroid $\boldsymbol{r}_{m}$ of the $m$ th microcube supporting the corresponding basis function ${ }^{1}$, for $m=1, \ldots, N$. This yields the MoM matrix equation $\mathbf{V}=\mathbf{Z I}$, where

$$
\begin{aligned}
\mathrm{Z}_{m n}= & \frac{1}{j \omega\left(\varepsilon-\varepsilon_{0}\right)}\left\langle\boldsymbol{f}_{n}(\boldsymbol{r}), \hat{\boldsymbol{p}} \delta\left(\boldsymbol{r}-\boldsymbol{r}_{m}\right)\right\rangle \\
& -\left\langle\boldsymbol{E}^{\mathrm{S}}\left(\boldsymbol{f}_{n}(\boldsymbol{r})\right), \hat{\boldsymbol{p}} \delta\left(\boldsymbol{r}-\boldsymbol{r}_{m}\right)\right\rangle, \\
\mathrm{V}_{m}= & \left\langle\boldsymbol{E}^{\mathrm{i}}, \hat{\boldsymbol{p}} \delta\left(\boldsymbol{r}-\boldsymbol{r}_{m}\right)\right\rangle .
\end{aligned}
$$

In the above equations, the scalar product $\langle\boldsymbol{a} \cdot \boldsymbol{b}\rangle=$ $\iiint_{\mathcal{V}_{\infty}} \boldsymbol{a} \cdot \boldsymbol{b} \mathrm{d} V$, and $\hat{\boldsymbol{p}} \in\{\hat{\boldsymbol{x}}, \hat{\boldsymbol{y}}, \hat{\boldsymbol{z}}\}$. The choice of the polarisation $\hat{\boldsymbol{p}}$ is similar to that of the micro-basis

\footnotetext{
${ }^{1}$ The collocation method is herein equivalent to Galerkin's method when evaluated through the mid-point integration rule.
}

function $\boldsymbol{f}_{n}$. Specifically,

$\boldsymbol{f}_{n}(\boldsymbol{r})= \begin{cases}\hat{\boldsymbol{x}}, & \boldsymbol{r} \in \mathcal{V}_{n}, n \in\{1, \ldots, N / 3\} \\ \hat{\boldsymbol{y}}, & \boldsymbol{r} \in \mathcal{V}_{n}, n \in\{N / 3+1, \ldots, 2 N / 3\} \\ \hat{\boldsymbol{z}}, & \boldsymbol{r} \in \mathcal{V}_{n}, n \in\{2 N / 3+1, \ldots, N\} \\ \mathbf{0}, & \text { elsewhere. }\end{cases}$

where $\mathcal{V}_{n}$ is the cubic support of $\boldsymbol{f}_{n}$ with edge length $a$ and volume $a^{3}$.

For non-overlapping basis functions, the field $\boldsymbol{E}^{\mathrm{S}}$, generated by $\boldsymbol{f}_{n}$ in (3a) closely resembles that of the dipole. For instance, for a $z$-oriented dipole with dipole moment $I l=a^{3}$ (since we have a uniform current of unit amplitude in each micro-cube), the radiated electric near field at $\boldsymbol{r}_{m}$ becomes

$$
\begin{aligned}
E_{x} & =\frac{a^{3} C_{m n}}{4 \pi j \omega \varepsilon_{0}} x z e^{-j k_{0}\left|\boldsymbol{r}_{m n}\right|}, \\
E_{y} & =\frac{a^{3} C_{m n}}{4 \pi j \omega \varepsilon_{0}} y z e^{-j k_{0}\left|\boldsymbol{r}_{m n}\right|}, \\
E_{z} & =\frac{a^{3}}{4 \pi j \omega \varepsilon_{0}}\left[C_{m n} z^{2}+\frac{k_{0}^{2}}{\left|\boldsymbol{r}_{m n}\right|} \ldots\right. \\
& \left.-\frac{j k_{0}}{\left|\boldsymbol{r}_{m n}\right|^{2}}-\frac{1}{\left|\boldsymbol{r}_{m n}\right|^{3}}\right] e^{-j k_{0}\left|\boldsymbol{r}_{m n}\right|},
\end{aligned}
$$

where $k_{0}$ is the free space propagation constant, $\omega$ is the angular frequency, $\boldsymbol{r}_{m n}=\boldsymbol{r}_{m}-\boldsymbol{r}_{n}$, and

$$
C_{m n}=-\frac{k_{0}^{2}}{\left|\boldsymbol{r}_{m n}\right|^{3}}+\frac{3 j k_{0}}{\left|\boldsymbol{r}_{m n}\right|^{4}}+\frac{3}{\left|\boldsymbol{r}_{m n}\right|^{5}} .
$$

The self-term $\mathrm{Z}_{n n}$, for $m=n$, can be computed analytically using the singularity subtraction method, by accounting for the static part of the Green's function only, and thus ignoring the electrodynamic residual part. After some mathematical manipulation, we obtain the approximation

$$
\mathrm{Z}_{n n} \approx \frac{a^{3}}{j \omega \varepsilon_{0}}\left[\frac{\varepsilon}{\left(\varepsilon-\varepsilon_{0}\right)}-\frac{4}{\pi} \arctan \left(\frac{1}{\sqrt{3}}\right)\right] .
$$

Once a well-conditioned moment matrix equation is constructed, we can solve for the unknown expansion coefficient vector $\mathbf{I}=\mathbf{Z}^{-1} \mathbf{V}$. The matrix fill and solution times, with respective time complexities of $\mathcal{O}\left(N^{2}\right)$ and $\mathcal{O}\left(N^{3}\right)$, can be reduced significantly by using the ACA and CBFM [4].

In CBFM, the problem is subdivided into smaller sub-blocks [see e.g. Fig. 2(b)]; each sub-block supports a higher level basis function ( $\mathrm{CBF}$ ) constructed from a lower-level group of micro-domain basis functions having predetermined fixed expansion coefficients. In CBFM, $\boldsymbol{J}_{\text {eq }}$ is therefore expanded as

$$
\boldsymbol{J}_{\mathrm{eq}}(\boldsymbol{r})=\sum_{n=1}^{P} I_{n}^{\mathrm{CBF}} \boldsymbol{J}_{n}^{\mathrm{CBF}}(\boldsymbol{r}),
$$




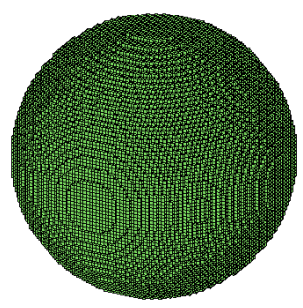

(a) 791361 micro-domain basis functions

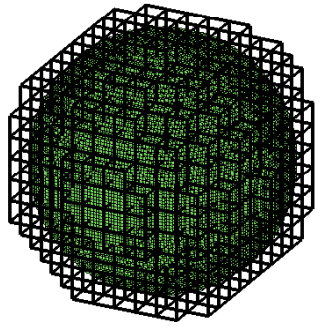

(b) 1728 CBF-blocks
Figure 2: Graphical illustration of the problem when subdivided by micro-cubes and CBF blocks.

where $P$ is the total number of CBFs employed for the problem; $\boldsymbol{J}_{n}^{\mathrm{CBF}}$ represents the $n$th $\mathrm{CBF}$ and $I_{n}^{\mathrm{CBF}}$ represents the $n$th unknown expansion coefficient for that $\mathrm{CBF}$. Each $\mathrm{CBF}$ is expanded in terms of micro-basis functions with known expansion coefficients $\left\{\alpha_{l}^{n}\right\}_{l=1}^{N}$, as; $\boldsymbol{J}_{n}^{\mathrm{CBF}}(\boldsymbol{r})=\sum_{l=1}^{N} \alpha_{l}^{n} \boldsymbol{f}_{l}(\boldsymbol{r})$, where most elements of the column vector $\boldsymbol{\alpha}^{n}=$ $\left[\alpha_{1}^{n}, \ldots, \alpha_{N}^{n}\right]^{T}$ are zero, since the CBF has a local support. To determine the nonzero entries, the corresponding block is first extended by a few neighbouring cells, then illuminated by a plane wave spectrum (PWS), truncated again to the original $\mathrm{CBF}$ block size. Following this, the number of CBFs generated is then reduced via the use of the Singular Value Decomposition (SVD) procedure with a threshold set on the singular values. Because of the use of the PWS approach, the CBFs can be used to solve the scattering problem for multiple incident angles without the need to generate them anew for each angle. Upon introducing the sparse $N \times Q$ column-augmented matrix $\mathbf{J}_{\mathrm{CBF}}=\left[\boldsymbol{\alpha}^{1}, \ldots, \boldsymbol{\alpha}^{Q}\right]$, where $Q$ is the total number of $\mathrm{CBFs}$, we arrive at the reduced moment matrix equation $\mathbf{Z}^{\mathrm{CBF}} \mathbf{I}^{\mathrm{CBF}}=\mathbf{V}^{\mathrm{CBF}}$, where

$$
\begin{aligned}
& \mathbf{Z}^{\mathrm{CBF}}=\mathbf{J}_{\mathrm{CBF}}^{\mathrm{T}} \mathbf{Z}_{\mathrm{CBF}}, \\
& \mathbf{V}^{\mathrm{CBF}}=\mathbf{J}_{\mathrm{CBF}}^{\mathrm{T}} \mathbf{v},
\end{aligned}
$$

and where the rank of $\mathbf{Z}$ is $Q$. This matrix equation can be solved rapidly for $\mathbf{I}^{\mathrm{CBF}}$ by using standard Gaussian elimination techniques. The ACA is used to construct $\mathbf{Z}^{\mathrm{CBF}}$ rapidly, since it exploits the rank deficiency of the off-diagonal block of the moment matrix $\mathbf{Z}$ by factorising it in lower-rank matrices. The effective rank is particularly small for distant groups of micro-basis functions, or CBFs.

\section{NUMERICAL RESULTS}

MatLab computations have been performed on a laptop computer equipped with a $2.2 \mathrm{GHz}$ dual core processor, and 2 GB of RAM. For the HFSS computations, a desktop server has been used equipped with an Intel Xeon E5345 quad core processor $(2.33 \mathrm{GHz})$, and $4 \mathrm{~GB}$ of RAM.

\subsection{Field Convergence}

Fig. 3 shows that, if a source cube containing a single equivalent dipole is subdivided into smaller sub-cubes, where each sub-cube contains a dipole with a corresponding smaller dipole moment, the accuracy of the computed $Z_{m n}$ does not increase significantly. Even for a single dipole, the field at the centroid of a direct adjacent cell can be computed sufficiently accurately.

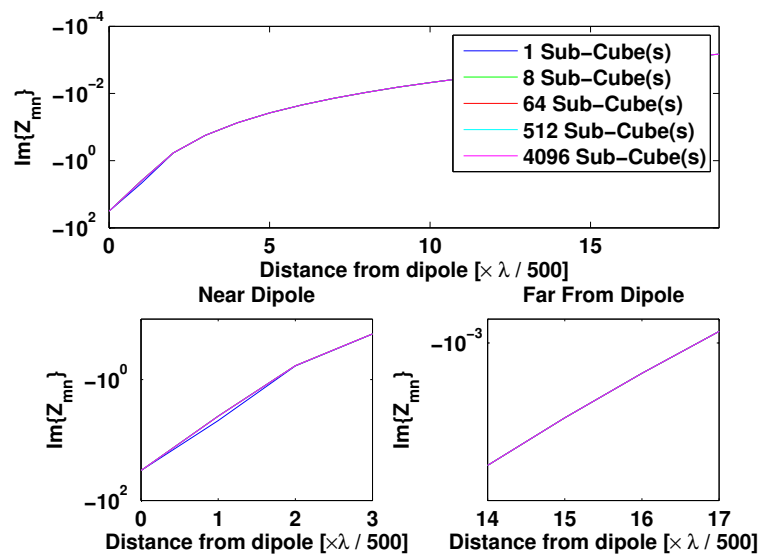

Figure 3: Imaginary part of $Z_{m n}$ for different subdivisions of the source cube basis functions.

\subsection{Effect of the Mesh Cell Size}

As shown in Fig. 4, the electric field can be modelled more accurately by reducing the support size of the micro-basis functions, especially for the fields in the interior region. The fields external to the dielectric object converge much faster.

\subsection{Scattering from a Dielectric Sphere}

A comparison between the MEDM, HFSS and the analytical Mie-series solution, is presented in Fig. 5 for a dielectric sphere of radius $\lambda /(4 \pi)$. For HFSS, if we use a max. delta energy of 1e-3 in 15 mesh refinement steps, it takes 35123 tetrahedra and a total simulation time of $28 \mathrm{~min}$. The MEDM employs 6309 micro-domain basis functions of edge length $\lambda / 100,8 \mathrm{CBF}$ blocks are used for the subdivision (3 cell extension), each employing only 12 CBFs (96 CBFs in total). The solve time for the problem is less than $150 \mathrm{sec}$. On average, the results shown in Fig. 5 demonstrate that the MEDM achieves a better accuracy as compared to that of the HFSS software. Although, the external scattered field has been determined very accurately, the internal field 

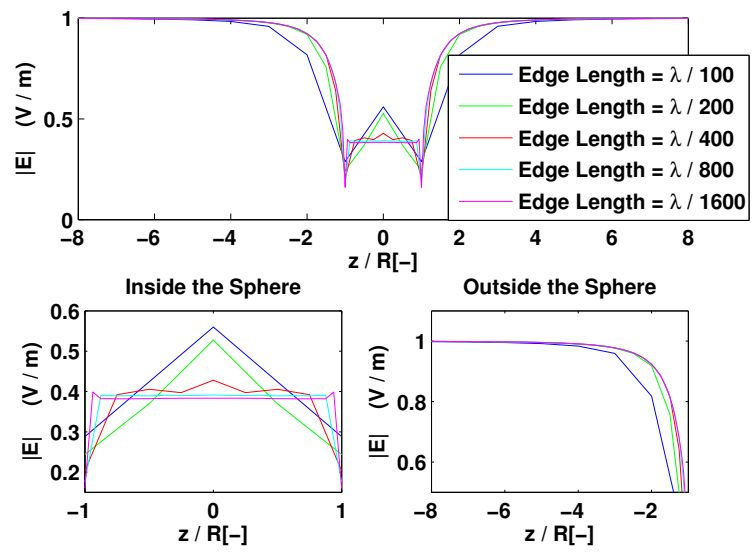

Figure 4: Magnitude of the electric field inside a homogeneous dielectric sphere along the $z$-axis; $\varepsilon_{r}=6$, $k_{0} R=\pi / 50$.

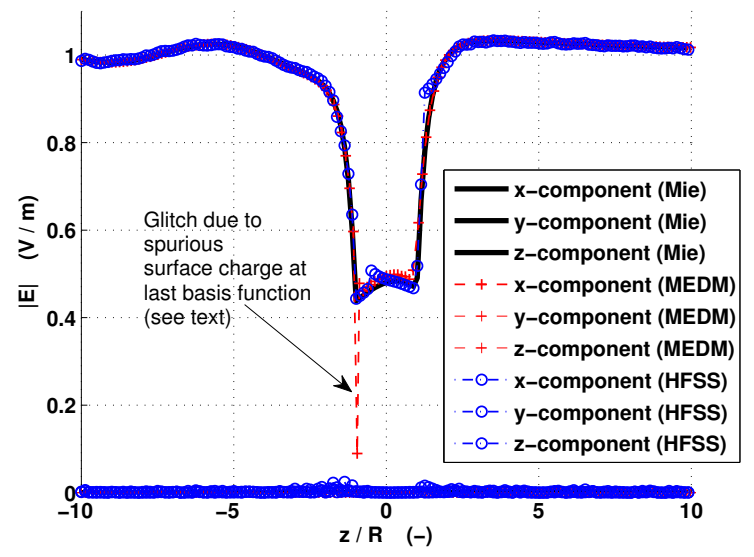

Figure 5: Magnitude of the electric field inside a homogeneous dielectric sphere along the $x$ axis; $\varepsilon_{r}=6$, $k_{0} R=1 / 2$

exhibits an anomalous glitch. This is attributed to the pulse-type of basis functions for the current, which gives rise to surface charges at the end faces of the cubic support that cannot be compensated for by an opposite charge of adjacent basis functions (if at the boundary of the object). Most often one is interested in physical quantities that are integrals over the fields or currents (scattering patterns, power losses, energy storage, etc.); hence, the glitch at only the last cell may not be of much concern. For instance, the time-average stored electric energy 1/4 $\iiint \varepsilon|\boldsymbol{E}|^{2} \mathrm{~d} V$ is for MEDM: $5.68 \mathrm{e}-15 \mathrm{~J}$; HFSS: 5.62e-15 J; and Mie: 5.77e-15 J, which shows that the MEDM result is closer to the analytically derived value than the one computed by HFSS. Nonetheless, to mitigate the problem of the glitch, research is currently ongoing to model both the current and charges by pulse-type basis functions, while weakening the hard constraint on the continuity equation for charges and currents at small scales. The latter would require us to add only $\mathrm{N} / 3$ additional micro-basis functions, since the charge density does not carry polarisation information because it is a scalar quantity.

\section{CONCLUSION}

Micro-domain basis functions have been employed to model the currents in dielectric bodies with high precision. The large number of independent basis functions is reduced significantly via the use of the CBFM, rendering the algorithm memory efficient, with little effect on the accuracy of the solution. Concurrently, the ACA algorithm has been used for rapid construction of the reduced moment matrix. In summary, the novel method proposed herein, and referred to as MEDM, is easy to implement, it is fast as well as memory efficient, and it can solve electrically large problems of arbitrary shape with relative ease. Future work will be devoted to develop methods to mitigate the effect of spurious glitches in the electric field, arising as a consequence of choosing pulse-type basis functions for the current while imposing a hard constraint on the continuity between surface currents and charges at small scales.

\section{Acknowledgments}

This work is part of the research programme $\mathrm{Ru}-$ bicon, which is partly financed by the Netherlands Organisation for Scientific Research (NWO).

\section{References}

[1] R. Maaskant et al., "Evaluation of the radiation efficiency and the noise temperature of low-loss antennas," IEEE Antennas and Wireless Propagation Letters, vol. 8, pp. 1166-1170, 2009.

[2] R. Mittra et al., "A universal dipole-momentbased approach for formulating MoM-type problems without the use of Green's functions," Proc. of the 4th Europ. Conf. on Antennas and Propagat. (EuCAP), 12-16 April, pp. 1-3, 2010.

[3] V. V. Prakash and R. Mittra, "Characteristic basis function mehod: A new technique for efficient solution of method of moments matrix equations," Microwave and Optical Technology Letters, vol. 36, no. 2, Jan. 2003.

[4] R. Maaskant, R. Mittra, and A. Tijhuis, "Fast analysis of large antenna arrays using the characteristic basis function method and the adaptive cross approximation algorithm," IEEE Trans. Antennas Propag., vol. 56, no. 11, 2008. 